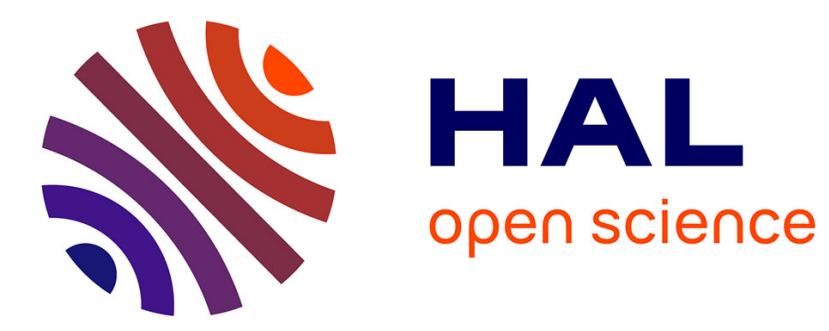

\title{
L'installation du Père de Foucauld dans l'Ahaggar
} Paul Pandolfi

\section{To cite this version:}

Paul Pandolfi. L'installation du Père de Foucauld dans l'Ahaggar. Journal des Africanistes, 1997, 67, pp.49 - 69. 10.3406/jafr.1997.1139 . hal-03182201

\section{HAL Id: hal-03182201 \\ https://hal.science/hal-03182201}

Submitted on 26 Mar 2021

HAL is a multi-disciplinary open access archive for the deposit and dissemination of scientific research documents, whether they are published or not. The documents may come from teaching and research institutions in France or abroad, or from public or private research centers.
L'archive ouverte pluridisciplinaire HAL, est destinée au dépôt et à la diffusion de documents scientifiques de niveau recherche, publiés ou non, émanant des établissements d'enseignement et de recherche français ou étrangers, des laboratoires publics ou privés. 


\section{L'installation du Père de Foucauld dans l'Ahaggar}

In: Journal des africanistes. 1997, tome 67 fascicule 2. pp. 49-69.

\section{Résumé}

En 1905, le Père de Foucauld s'installe à Tamanrasset, au cœur même du massif de l'Ahaggar. À l'encontre du discours hagiographique qui domine largement les nombreuses biographies foucaldiennes, l'examen de documents d'archives jusqu'alors inexploités démontre que cette installation ne fut rendue possible que par le rapport de force alors établi entre les autorités coloniales françaises et les Kel-Ahaggar après la défaite militaire de ces derniers au combat de Tit (1902).

\section{Abstract}

In 1905, Father de Foucaul began living at Tamanrasset, in the heart of the Ahaggar mountain range. A study of heretofore unexplored archival material shows that this installation was made only possible by the power relationships existing at the time between the French colonial authorities and the Kel-Ahaggar followings the latter's military defeat at the battle of Tit (1902). This conclusion runs counter to the hagiographie discourse which dominates in de Foucauld's many biographies.

Citer ce document / Cite this document :

Pandolfi Paul. L'installation du Père de Foucauld dans l'Ahaggar. In: Journal des africanistes. 1997, tome 67 fascicule 2. pp. 4969.

doi : 10.3406/jafr.1997.1139

http://www.persee.fr/web/revues/home/prescript/article/jafr_0399-0346_1997_num_67_2_1139 


\section{Paul PANDOLFI*}

\section{«Sauront-ils séparer entre les soldats et les prêtres? »" L'installation du Père de Foucauld dans l'Ahaggar}

\section{Résumé}

En 1905, le Père de Foucauld s'installe à Tamanrasset, au ccur même du massif de l'Ahaggar. À l'encontre du discours hagiographique qui domine largement les nombreuses biographies foucaldiennes, l'examen de documents d'archives jusqu'alors inexploités démontre que cette installation ne fut rendue possible que par le rapport de force alors établi entre les autorités coloniales françaises et les Kel-Ahaggar après la défaite militaire de ces derniers au combat de Tit (1902).

Mots-clefs

Touareg, Dinaux, Foucauld, Mûsa agg Amastân, colonisation.

\section{Abstract}

In 1905, Father de Foucaul began living at Tamanrasset, in the heart of the Ahaggar mountain range. A study of heretofore unexplored archival material shows that this installation was made only possible by the power relationships existing at the time between the French colonial authorities and the Kel-Ahaggar followings the latter's military defeat at the battle of Tit (1902). This conclusion runs counter to the hagiographic discourse which dominates in de Foucauld's many biographics.

\section{Key-words}

Tuareg, Dinaux, Foucauld, Mûsa agg Amastân, colonisation.

\footnotetext{
* Centre d'Anthropologie Généralisée, Université de la Réunion, B.P. 7151, 97715
} Saint-Denis Messag Cédex 9. 


\section{LE DISCOURS HAGIOGRAPHIQUE}

Juin 1905 : Pour la seconde fois de sa vie, le Père de Foucauld parcourt l'Ahaggar en compagnie d'une colonne militaire commandée par le capitaine Dinaux. Déjà, l'année précédente il avait suivi le commandant Laperrine lors d'une importante tournée qui a conduit ce dernier jusqu'à Timiaouine (mars-juin 1904) puis le lieutenant Roussel dans la Tefedest et le nord de l'Atakor (juin-septembre 1904)' ${ }^{1}$.

Mais, à aucun moment, il n'a pu alors rencontrer Mûsa agg Amastân qui, depuis janvier 1904, a été nommé amenûkal [chef suprême de l'Ahaggar] par les autorités coloniales françaises. Ce n'est que le 25 juin 1905 qu'a lieu, à In Ouzel, cette rencontre tant attendue entre la colonne Dinaux et le chef Kel-Ahaggar. Dans une récente biographie consacrée à Foucauld, ce moment capital est ainsi présenté (Antier 1997: 213):

«En grande pompe, devant le capitaine Dinaux, Moussa fit sa soumission, et amende honorable pour ses exactions passées. [...] Puis Dinaux lui présenta Charles de Foucauld, comme "le marabout chrétien serviteur du Dieu unique", qui aimait la solitude et le désert et souhaitait étudier la langue et la culture des Touareg. Moussa le prit aussitôt sous sa protection et accepta de le voir s'installer au Hoggar. Ne serait-il pas, au pire, une sorte d'otage des Touareg, au mieux un gage de paix durable ? Bien conscient de ces calculs, Charles voyait se réaliser son rêve de créer un ermitage au cœur du Sahara où le Christ était ignoré. On prit rendez-vous pour le ler août à Abalessa, d'où Moussa le conduirait à Tamanrasset, qui semblait le village le mieux placé, au carrefour des routes du désert, pour qu'il exerce son apostolat silencieux et apprenne à connaître le peuple Kel Ahaggar. »

Dans une telle présentation, tout semble réglé dès le début. Certes plane encore une interrogation sur les véritables intentions de Mûsa mais il ne fait cependant aucun doute qu'entre les deux hommes un courant de sympathie et d'estime réciproque s'est d'emblée instauré. De même, l'installation de Foucauld dans l'Ahaggar ne paraît poser aucun problème et, à en croire le récit ci-dessus, ce fut là une formalité qui put très rapidement trouver solution. Or, cette (re)écriture de la rencontre d'In Ouzel se retrouve, à quelques variantes près, dans la quasi-totalité des nombreux ouvrages consacrés au Père de Foucauld. Cette exposition des faits est très souvent "légitimée " par des écrits de Foucauld lui-même. Ainsi est-il souvent fait référence - à ce moment-là - aux lignes consacrées dans son diaire au "portrait 》 de Mûsa (Foucauld 1993 : 178) :

« Mousa très bien, très intelligent, très ouvert, très pieux musulman, voulant le bien en musulman, libéral, mais en même temps ambitieux, et aimant argent et

\footnotetext{
' Voir Laperrine (1905), Roussel (1904), Foucauld (1993 et 1994-95).
} 
plaisir et honneur [...] Il est tout dévoué à Bei (d'Attalia) de qui il dit avoir tout reçu et qu'il déclare aimer plus que ses yeux. En résumé, Mousa est un bon et pieux musulman, ayant les idées et la vie, les qualités et les vices d'un musulman logique, et ayant en même temps l'esprit aussi ouvert que possible. "

La technique d'écriture adoptée par la plupart des biographes de Foucauld intègre ces lignes célèbres au récit - plus ou moins romancé de la rencontre d'In Ouzel, créant ainsi un télescopage entre un événement daté ( 25 juin 1905) et un écrit qui lui est postérieur puisque datant au mieux du 20 juillet 1905 . Ce qui est ainsi mis entre parenthèses est la période passée entre In Ouzel et la rédaction de ces lignes par Foucauld. Dès lors, le jugement (plutôt) positif porté sur Mûsa semble une véritable "révélation »: le chef Kel-Ahaggar serait apparu tel à Foucauld dès leur première rencontre. De plus, après ces lignes consacrées à Mûsa, figure dans nombre d'ouvrages ${ }^{2}$ une autre phrase : «D'accord avec Mousa, mon installation au Hoggar est décidée » (Ibid.). Notation inscrite, tout comme le portrait précédemment cité, à la date du 4 juillet 1905 dans le diaire de Foucauld. Mais une lecture attentive du texte montre qu'à l'évidence la rédaction de cet assez long passage est bien postérieure à cette date. Foucauld y fait en effet référence à des événements datant du 10, voire du 19 juillet 1905 . Or, il ne s'agit pas là d'un simple problème de datation. Si le portrait de Mûsa et, plus encore, la notation concernant l'installation prochaine de Foucauld remontent au moins au 20 juillet 1905, cela tempère quelque peu l'impression d'immédiateté qui ressort de la plupart des lignes consacrées à cet événement. L'établissement de Foucauld, chrétien français introduit (de fait) par une colonne militaire, ne pouvait être un événement banal en cette période. Aussi cette installation n'a (peut-être) pas été aussi facile que le laisse supposer une écriture de type hagiographique qui, elle, a besoin, dès le départ, de cette symbiose quasi instantanée entre l'ermite et ses hôtes.

Foucauld lui-même nous fournit d'autres renseignements, mais ils sont finalement assez vagues en ce qui concerne la chronologie. Dans son diaire, à la date du 11 août 1905, figurent ces lignes maintes fois citées (Foucauld 1986:48):

« Par la grâce du divin Bien-Aimé Jésus, il m'est possible de m'installer, me fixer à Tamenghasset ou dans tout autre point du Hoggar, d'y avoir maison et jardin, et de m'y établir pour toujours... Cette possibilité me paraît même une volonté du Bien-Aimé... Je choisis Tamenghasset, village de 20 feux en pleine montagne, au cœur du Hoggar et des Dag-Ghali sa principale tribu, à l'écart de tous les centres importants: il ne semble pas que jamais il doive y avoir garnison, ni télégraphe, ni européen, et que de longtemps il n'y aura pas de mission : je choisis ce lieu délaissé et je m'y fixe... »

\footnotetext{
${ }^{2}$ Exemples parmi d'autres: Bazin $(1921: 310)$ et Vignaud $(1943: 207)$.
} 
Texte important puisque la localisation du futur ermitage de Foucauld y est clairement donnée. On remarquera cependant que les premières lignes de ce passage semblent indiquer que la possibilité de s'installer dans l'Ahaggar semble récente. Mais il est un autre témoignage qui, sur ce sujet, peut nous apporter quelque lumière : celui du capitaine Dinaux.

\section{LE TEMOIGNAGE DU CAPITAINE DINAUX}

Ce dernier était en effet à la tête de la tournée militaire avec laquelle, depuis mai 1905, Foucauld sillonnait le Sahara central. Le témoignage de cet officier a d'ailleurs été utilisé par certains biographes du Père de Foucauld. Il est notamment cité par M. Carrouges (1963 : 215) et J.-F. Six (1962: 145). Mais ces auteurs se référent à un écrit de Dinaux bien postérieur aux faits puisqu'il s'agit d'un article paru en 1946 (Dinaux 1946) et qui s'inscrit dans l'abondante littérature qui glorifie alors le rôle joué par Foucauld au Sahara central. Ces réserves seraient cependant de peu d'intérêt si on ne disposait du même capitaine Dinaux d'autres témoignages. Or, tel n'est pas le cas. Suite à l'importante tournée qu'il effectua dans l'Ahaggar de mai à octobre 1905, cet officier rédigea en effet un rapport dont une version abrégée fut publiée dans le Bulletin du Comité de l'Afrique Française de janvier-février et mars 1907. Ce rapport, qui avait pour but de rendre compte à ses supérieurs hiérarchiques du déroulement de sa tournée et des résultats politiques obtenus, a pour base un journal de bord que ce même officier a tenu, au jour le jour, tout au long de sa mission. Ce dernier document, intitulé "Carnet de renseignements politiques », est joint, en tant qu'Appendice $\mathrm{n}^{\circ} \mathrm{IV}$, au rapport de tournée consultable au Centre des Archives d'OutreMer d'Aix en Provence. Cependant, à la différence des autres appendices, il n'a pas été transmis aux différents ministères concernés comme le signale explicitement une annotation manuscrite (en date du 27 juin 1906) sur sa première page: «L'appendice $n^{\circ} 4$ n'a pas été copié ni transmis aux différents ministères. " En préambule, Dinaux précise d'ailleurs que « ce carnet n'intéresse que l'Annexe d'In Salah et cet exemplaire est le seul qu'elle possède ».

En résumé, nous possédons trois documents contemporains de cette période historique et signés par Dinaux lui-même :

a) le Carnet de renseignements politiques (Dinaux 1907b), à propos duquel Dinaux signale qu'il "a été tenu au jour le jour pendant la tournée » et que "les renseignements importants en ont été extraits pour servir à la rédaction du rapport d'ensemble de la tournée ».

b) le rapport de tournée rédigé par Dinaux (1907a).

c) la version abrégée de ce rapport parue dans le Bulletin du Comité de l'Afrique Française (Dinaux 1907c) et donc rendue publique. 
Malgré son indéniable valeur historique, ce dernier texte sera peu utilisé dans la suite de ce travail et ce pour une raison simple : à aucun moment il n'y est fait mention de la présence de Foucauld dans la tournée Dinaux et à fortiori de son installation à Tamanrasset. Autocensure significative: dans les conditions politiques de l'époque (et notamment le conflit ouvert entre cléricaux et anticléricaux), la présence de Foucauld dans les tournées militaires entreprises au Sahara central est un véritable " coup de force » de Laperrine. C'est lui et lui seul qui en a ainsi décidé à l'insu tant de ses supérieurs militaires que des politiques ${ }^{3}$. D'où cette disparition de Foucauld dans la version publique du rapport Dinaux ${ }^{4}$. En 1904 de même, la présence de Foucauld fut absolument passée sous silence dans le rapport du lieutenant Roussel. Par contre, dans le rapport de tournée établi par Dinaux, il est plusieurs fois fait référence au Père de Foucauld et une partie de ce document est d'ailleurs intitulée : "Installation du Père de Foucauld ».

Mais c'est bien le Carnet de renseignements politiques qui fournit des informations de première importance sur cette période où se concrétisèrent les projets d'installation de Foucauld dans l'Ahaggar. Dans ce document manuscrit de 103 pages, la présence et les projets de Foucauld sont mentionnés dans 7 passages de longueur et d'importance inégales. Certaines de ces notations sont très rapides. Ainsi apprend-on que, le 8 juillet 1905, dans le campement du chef des Iouarouaren «le Père de Foucauld soigne depuis 3 jours une jeune fille syphilitique " ou encore que, le 17 juillet, le chef Taytoq, Sidi agg Akaraji, " souffre d'une vieille blessure au genou et le Père de Foucauld le badigeonne à la teinture d'iode ". Ainsi, le journal de Dinaux remet en quelque sorte de l'ordre dans la vision que l'on peut avoir après-coup de cette tournée. Les objectifs principaux qui étaient fixés à cette dernière étaient de première importance. Selon Dinaux lui-même (1907a), il s'agissait de « rappeler à Moussa son rôle de chef soumis, [...] dire aux djemaâs ce que nous voulons et les conditions de leur soumission, $[\ldots]$ en un mot rendre la situation nette et le commandement plus facile ». Dans ce cadre, le

\footnotetext{
${ }^{3}$ Laperrine était coutumier du fait. Nombre d'initiatives majeures prises alors au Sahara central (raid Cottenest, projet de rejoindre Tombouctou en 1904, tournée vers Taouedenni en 1906...) le furent sans accord préalable de ses supérieurs hiérarchiques. Ceux-ci se retrouvant dès lors placés devant des "faits accomplis". Sur ce point, Foucauld jouera le jeu de Laperrine en demandant à ses proches de garder le plus strict silence. Voir, par exemple, sa lettre en date du 17-06-1904 à H. de Castries (1938 : 152). Cette politique du fait accompli est alors une constante de la politique impulsée par nombre d'officiers coloniaux en divers endroits de l'empire français: voir Ambler (1966), cité par Anderson (1996: 156).

${ }^{4}$ Voir aussi Gautier (1906). Ce dernier faisait également partie de la tournée Dinaux (du moins dans sa première partie) mais, dans l'intéressant compte rendu de son voyage paru dès 1906, il ne mentionne pas la présence de Foucauld. A contrario, voir Gautier (1931 : 39-63).
} 
projet d'installation du Père de Foucauld dans l'Ahaggar, projet réel et soutenu par Laperrine et des officiers comme Dinaux, ne peut apparaître à ce moment-là que comme un objectif très secondaire. Restent cependant des passages plus importants dans le journal de Dinaux. Premier exemple, ces lignes datées du 26 juin 1905 :

«Le soir, à 4 heures, débouchent en même temps devant le camp, la députation de Moussa venant du Nord et celle de Sidi ag Keradji venant du Sud [...] Palabre : la paix; suppression des razzias; bon voisinage; indépendance des Taïtoq avec Sidi; notre manière de commander; notre pénétration lente et progressive. La conversation dévie vers la religion, les chefs disant : nous obéirons en tout, mais à condition de ne pas changer de religion. Ce que nous avons fait en Algérie pour l'islamisme; mosquées, traitement, pèlerinage, ramadan... etc. Et la présence du Père de Foucauld pouvant être mal interprétée, on fait son éloge; ses deux grands principes: aidez-vous les uns les autres; rendez le bien pour le mal. Moussa riposte : cela est aussi dans notre religion." "

Nous sommes là au second jour de la fameuse entrevue d'In Ouzel. La veille, la rencontre tant attendue a eu lieu. Mûsa agg Amastân, monté sur un cheval et escorté par une délégation de seize Kel-Ahaggar, s'est présenté au capitaine Dinaux. Un premier palabre s'est alors déroulé.

Bien évidemment, Dinaux rapporte en détail tous ces événements dans son journal. Si l'on s'en tient à ce témoignage direct, à aucun moment le problème Foucauld ne fut alors évoqué. Ce dernier, nous le savons, est présent mais tout semble indiquer que ses projets d'installation dans l'Ahaggar n'ont pas été abordés. Ce 26 juin, il en fut de même, semble-t-il, si l'on se réfère au journal de Dinaux. Bien des problèmes autrement urgents et importants doivent être discutés et réglés. Et ce n'est finalement que parce que « la conversation dévie vers la religion ", que la présence (et non le projet) du Révérend Père est abordée par les militaires français eux-mêmes. En actes et paroles, c'est bien par les militaires français que Foucauld est introduit auprès des principaux chefs touaregs. Et l'on voit mal (au nom de quelle mystérieuse raison ?), comment ces derniers auraient pu, à ce moment-là du moins, opérer une séparation entre ces militaires et le prêtre ? L'éloge de Foucauld ne semble d'ailleurs que peu impressionner Mûsa qui, par sa réponse (ou plus exactement sa riposte selon le terme employé par Dinaux), indique bien qu'il campe dans sa propre religion, et ce d'autant plus que les arguments mis en avant ne constituent en rien une différence notable.

On relèvera également l'importance du fait religieux, qui reste aux yeux des Kel-Ahaggar le seul point non négociable. Un poncif très fréquent consiste à présenter les Touaregs comme des musulmans «superficiels» et «peu convaincus ${ }^{5}$. Les propos rapportés par Dinaux

\footnotetext{
${ }^{5}$ Depuis le XVIII ${ }^{\mathrm{e}}$ siècle au moins, la référence à l'islam était chez les Kel-Ahaggar un élément politique fondamental comme le démontre l'historique mouvementé de la lutte
} 
démontrent, si besoin est, le peu de validité de ce type de proposition. Mais cela signifie aussi que Foucauld cumule aux yeux des Kel-Ahaggar deux handicaps majeurs: celui d'être considéré comme un militaire français et comme le représentant d'une autre religion.

\section{UNE DIFFICILE INSTALLATION}

Voila un homme qui arrive chez eux au sein d'une colonne militaire et arborant des signes extérieurs particuliers.

«... il était simplement vêtu de la gandoura blanche, serrée à la taille par une ceinture de cuir à laquelle pendait un chapelet à gros grains noirs; pour coiffure, il portait une large chéchia à manchon de toile blanche garnie d'un couvrechef... $\|^{6}$

Son étrange bagage comprenait «... une légère tente de toile pour la célébration de la messe ; les objets indispensables au culte; un flacon de vin de messe dont il n'usait que par gouttes ; une table pliante pour servir d'autel ». Tôt le matin, il se faisait réveiller, "montait rapidement sa tente et disait sa messe »(Dinaux 1946).

$\mathrm{Si}$, comme le rappelle justement Sahlins (1989), «il n'est pas d'immaculée perception ", on voudra bien reconnaître qu'il y avait là de quoi soulever nombre d'interrogations sur la nature et la finalité du projet foucaldien. En deça des discours et des tentatives d'explication demeure ce face à face initial qui ne pouvait qu'engendrer chez les KelAhaggar un difficile questionnement ${ }^{p}$. Exemple significatif: lors de sa première tournée dans l'Ahaggar (1904), sur un petit carnet-agenda,

pour le pouvoir au sein de cette confédération (Pandolfi 1997). Mais cette référence était également présente dans la "vic mentale" des Kel-Ahaggar (Morel 1947) et, à cet égard, l'imprégnation islamique des poésies touarègues recueillies par Foucauld est particulièrement révélatrice (Kergoat 1979 et Merad 1976).

${ }^{6}$ Plusieurs clichés photographiques témoignent de la tenue alors arborée par le Père de Foucauld. Parmi ceux-ci figurent les clichés sur lesquels on voit deux Kel-Ahaggar simuler un duel avec boucliers et takuba. Le Père y assiste à l'arrière-plan. Ces photographies plusieurs fois reproduites, notamment dans le livre de Benhazera (1908: 19-22) et, en une version agrandie, dans les Cahiers Charles de Foucauld $\left(1948, \mathrm{n}^{\circ} 8\right)$, ont été prises par Laperrine lors de sa tournée de 1904. Par une lettre de Laperrine à Cauvet (18/06/1904), on apprend que les deux protagonistes de ce duel simulé n'étaient autre qu'Aziouel ag Seghada (chef des Kel-Ahnet) et son frère

${ }^{7}$ Voir la lecture faite par G. Lenclud des travaux de Sahlins consacrés à l'arrivée de Cook aux Iles Fidji : "Deux emics se font face. Avant que d'échanger des émissaires, des gestes ou des paroles, [...] deux cultures soumises à l'expérience de la rencontre échangent d'abord des regards. [...] Or il est assez bien connu que n'importe quel Ego culturel ne peut voir l'Autre culturel, et quoi que ce soit de cet Autre, qu'à partir de ses catégories. [...] Ces histoires offrent un spectacle fascinant, en temps presque réel, celui d'un ordre de catégories culturelles arraché à sa routine, sorti de sa quiétude structurale, mis en demeure d'intégrer l'inconnu, de le reconnaître » (1991: 52). 
Foucauld inscrit des phrases en tamâhaq afin de pouvoir assurer un minimum de conversation avec les nomades qu'il rencontre. $Y$ figure celle ci : " Akatab ouader oul foul takarbest in enta as lemeder messinar d iklan nit : Ce cœur écrit sur ma robe, il est là pour que je me souvienne de Dieu et des hommes (pour les aimer). " Comme le relève justement $M$. Serpette (1997: 40), "Foucauld, pas encore assuré de son touareg, écrivait par avance une réponse à une question qui devait lui être souvent posée... ${ }^{8}$ Dans la suite du journal de Dinaux, Foucauld ne réapparaît vraiment que le 15 juillet 1905. Ce jour-là, Mûsa a demandé à l'officier français l'autorisation de quitter la colonne pour se rendre à ses campements et promis de la rejoindre à nouveau trois semaines plus tard à Abalessa.

Cela fait donc environ vingt jours que Dinaux et Foucauld fréquentent le chef Kel-Ahaggar. La possibilité d'une installation a-t-elle été évoquée ? Peut-être, sûrement même, mais le journal reste muet sur ce point. Par contre, à cette date du 15 juillet, Dinaux se montre prolixe et couvre cinq pages manuscrites de son carnet. Dans cette longue notation, figure ce passage :

"Sur la demande du Père de Foucauld, je demande à Moussa comment les gens du Hoggar accepteraient l'installation du Père dans leur pays et s'il ne pourrait en résulter d'ennui pour lui. Je lui fais remarquer que le Père soignera les malades, répandra des graines de légumes et des plantes d'arbres, étudiera les mœurs et les besoins des gens et sera à même de nous fournir les plus utiles renseignements sur le bien à faire dans le pays. J'ajoute que le Père est pour nous ce qu'est Bey pour eux, et que les moindres contrariétés qui lui seraient faites prendraient de grosses proportions. Moussa répond très franchement qu'il est rallié à ce projet qu'il approuve et que l'an dernier il a blâmé Sughi pour avoir conseillé au colonel d'attendre encore à ce sujet. Il comprend ce que nous voulons et saura, dit-il, l'imposer à ses gens. "

Le ton même de la première phrase tout comme l'emploi du conditionnel sont ici révélateurs: à cette date (15-07-1905) l'installation éventuelle du Père de Foucauld dans l'Ahaggar est loin d'être acquise. On notera également que, selon ces notes, ce sont les militaires français qui, sur ce point, s'adressent à Mûsa, c'est l'officier responsable de cette tournée qui, "sur la demande du Père de Foucauld », s'inquiète de l'effet que pourrait avoir l'installation de ce dernier au cour du Sahara. Quand aux arguments avancés, ils sont eux aussi significatifs.

\footnotetext{
${ }^{8}$ Antoine Chatelard (in litt. 16-05-1997) nous fait remarquer que «cette phrase de très mauvais Tamahaq révèle cette préoccupation initiale de Charles de Foucauld qui veut s'exprimer et tout expliquer. Il ne pense même pas à noter la question telle que les gens pouvaient la lui poser. Il ne donne que la réponse ". Par la suite, après le séjour de Motylinski dans l'Ahaggar en 1906, s'ouvrira une nouvelle perspective : "Il ne s'agit plus de traduire pour dire aux Touaregs ce qu'il veut leur dire, mais d'écouter ce qu'ils ont à dire » (Chatelard $1995: 175)$.
} 
Le 26 juin 1905, c'est sur le terrain des principes religieux que s'était opéré l'éloge de Foucauld. Dorénavant, ce sont des intérêts bien plus pratiques (médecine et agriculture notamment) qui sont mis en avant. Quand l'aspect "religieux » est abordé, il se moule dans un cadre de référence bien connu des Kel-Ahaggar : Foucauld est alors explicitement comparé à Bây al-Kunti. Ce célèbre religieux, depuis sa zâwiya de Téleya dans l'Adagh des Ifoghas, jouait un rôle de première importance dans l'ensemble du Sahara central et surtout était le maître spirituel de Mûsa'. Plus exactement encore en précisant que Foucauld est pour les militaires français ce que Bây est pour les Touaregs l'on passe à une menace directe et parfaitement compréhensible pour les interlocuteurs de Dinaux. Si Foucauld occupe une telle position cela signifie aussi « que les moindres contrariétés qui lui seraient faites prendraient de grosses proportions. 》

La réponse de Mûsa est, elle aussi, très significative. Nous nous attarderons plus particulièrement ici sur sa dernière phrase telle qu'elle est rapportée par Dinaux. L'emploi de la première personne du pluriel semble indiquer que pour lui le projet de Foucauld est aussi (surtout?) celui des autorités militaires françaises. La dernière proposition laisse au moins planer un doute sur le désir et la volonté des Kel-Ahaggar de voir un akafar ${ }^{10}$ s'installer dans l'Ahaggar. D'où aussi l'engagement de Mûsa d'user de toute son influence voire de son pouvoir pour l'imposer aux récalcitrants.

Dans le carnet de Dinaux, les autres notations concernant Foucauld se trouvent consignées aux dates du 13 et 16 août 1905 . Le 13 août est justement le jour où Mûsa retrouve la colonne Dinaux à Tamanrasset ${ }^{11}$. Par rapport à la notation précédente un fait est d'ores et déjà acquis : le Père de Foucauld s'installe dans l'Ahaggar à Tamanrasset : "Moussa apprend avec plaisir que le Père de Foucauld a choisi Tamanghasset pour lieu de résidence; il répète à plusieurs reprises que nous sommes chez nous et que le Père est le bienvenu. " Ce choix a du très certainement s'opérer début août 1905. Sur ce point, deux écrits de Foucauld lui-même nous fournissent des repères. Outre le passage déjà cité de son diaire en date du 11 août 1905, jour de l'arrivée à Tamanrasset, existe également

\footnotetext{
${ }^{9}$ Fidèle en cela à la politique de "collaboration » suivie par d'importantes confréries (Tidjaniya et Quadiriya notamment), Bây a toujours prôné une attitude pacifiste envers la pénétration française. Dès le tout débuts de leur installation au Sahara, les militaires français auront recours à l'influence exercée par Bây sur les populations locales (voir Marty 1920, I : 116-122 ; Richer 1924).

${ }^{10}$ Dans l'Ahaggar, par ce terme (pl. ikufar) sont désignés les Européens. Mais, dérivé de l'arabe kafir, ce mot a également une forte connotation religieuse : un akafar est aussi (surtout?) un infidèle.

${ }^{11}$ Mûsa avait quitté la colonne Dinaux le 15 juillet 1905 afin de se rendre dans des campements situés aux environs d'In Ouzel. Il était convenu qu'il la retrouverait 20 jours plus tard à Abalessa. En fait, ayant été retardé - selon ses dires - par des pluies abondantes, il rejoignit Dinaux le 13 aout à Tamanrasset.
} 
une lettre adressée, depuis Ennedid, à Mme de Bondy le 6 août 1905 . Dans cette correspondance il est notamment écrit (Foucauld 1966: 138):

"Je vais m'établir pour quelques mois, peut-être plus, dans cette région, au village de Tamanrasset... J'avais cherché cet établissement l'été dernier, sans réussir ; cette année, il semble que je le puisse ; je crois devoir profiter de cette possibilité, de cette occasion, ne pas laisser se fermer cette porte entrouverte... Selon toute probabilité, je vais donc m'établir là pour au moins l'été, l'automne, l'hiver, peut-être pour beaucoup plus; je m'installe sans faire de projets. $\gg{ }^{\prime 2}$.

Ce choix est présenté dans le journal de Dinaux comme une décision de Foucauld lui-même. Il est cependant fort probable que Mûsa l'ait orienté dans le choix de ce lieu. Comment pourrait-il en être autrement dans la mesure où ni Foucauld ni Dinaux ne connaissaient Tamanrasset ${ }^{13}$ et que le choix de ce site est évoqué par Foucauld avant même que la colonne Dinaux y soit parvenue ? Cette influence de Mûsa est d'ailleurs souvent relevée dans la littérature consacrée au Père de Foucauld. Il est alors fait référence au texte publié en 1946 par le général Dinaux (Dinaux 1946:42):

" Au cours de nombreux entretiens nous examinâmes le côté pratique de la question, et le choix de la résidence du Père s'arrêta sur Tamanrasset. C'était le point d'attache de la tribu serve des Dag Rali, la plus nombreuse et la plus fidèle à Moussa ; il y avait un centre de cultures et une petite colonie de Harratine du Tidikelt, que visitaient souvent les caravanes; enfin le climat était tempéré. Devant l'adhésion de Moussa et sa promesse solennelle de répondre de la sécurité du Père sur sa tête, celui-ci prit irrévocablement la décision de s'installer définitivement à Tamanrasset à notre retour de l'Adrar des Iforas, et de grand cœur je lui en accordai l'autorisation. »

Tout indique que le "nous" ici utilisé englobe non seulement Foucauld et Dinaux mais aussi Mûsa et les autres notables Kel-Ahaggar alors présents. Si la décision finale revient à Foucauld, c'est à partir des renseignements fournis par ces derniers qu'elle est prise.

\footnotetext{
${ }^{12}$ A la même date, dans une lettre adressée à Mgr Guérin, Foucauld écrit : « Je vais très probablement m'établir à Tamanrasset, village au cœur du Hoggar [...] tout bien réfléchi, je préfère Tamanrasset, assez central et cependant en dehors des lieux qui pourront recevoir des postes militaires ou être sur le passage d'une ligne télégraphique. »

${ }^{13}$ Avant le séjour de Dinaux en ce lieu, étaient rapidement passés par Tamanrasset : le raid Cottenest ( 6 mai 1902), la veille même du combat de Tit, et le "contre-rezzou " Guillo-Lohan (14 novembre 1902). Ce dernier officier présentait ainsi ce centre dans son rapport de tournée(Guillo-Lohan 1903).: "Une heure de marche nous amène dans la vaste plaine de reg où coule l'oued Tamanrasset, à sa sortie de la Koudia. Les maisons du village, quatre ou cinq cahutes en terre et dix-huit zéraibs y sont éparses ça et là, entourées d'assez belles cultures qui appartiennent : moitié aux Ouled-Rali, moitié aux Dag-Ouan-Taheli. La création de ce centre remonte à une quinzaine d'années... »
} 


\section{LES RETICENCES DES DAG-GHALI}

Mais l'écriture hagiographique s'en tient généralement là. Or, il se trouve que le premier argument cité en faveur de Tamanrasset, à savoir que ce centre est «le point d'attache de la tribu serve des Dag Rali, la plus nombreuse et la plus fídèle à Moussa ", se retrouve quelques temps plus tard cité à l'appui d'une tentative contraire de Mûsa. À la date du 16 août, on peut en effet lire dans le journal de Dinaux les phrases suivantes :

« Au sujet de l'installation du Père, Moussa fait ce qu'il peut pour déterminer le Père à choisir Endid plutôt que Tamanghasset, qui est le centre d'attache des Dag Rali. Sur la question d'eau pour la culture d'un jardin potager, il préconise le creusement d'une foggara de moitié entre le Père et lui, plutôt que l'achat d'une part d'eau qui, dit-il, nuirait beaucoup aux travailleurs. "

Il est intéressant de replacer ces apparentes volte-face de Mûsa dans le contexte de l'arrivée de la tournée Dinaux en territoire Dag-Ghâli. Quand, le 11 août 1905, la tournée arrive à Tamanrasset, les Dag-Ghâli (et notamment leur chef Dua agg Ag-Iklan) lui réservent un accueil plutôt froid comme le relève Dinaux dans son journal :

«Doua se présente dans l'après-midi avec quelques Dag Rali ; son attitude est bizarre, c'est celle d'un condamné ; il est vêtu misérablement et paraît visiblement contrarié de la démarche qu'il fait, il n'a osé s'y dérober cependant. Il est peu loquace et je n'arrive pas à le mettre en confiance." "

Si le lendemain les Dag-Ghâli se montrent plus avenants, Dinaux en précise la raison. Mûsa, qui est en route pour Tamanrasset, leur a dépêché un émissaire. Et Dinaux, sans illusion, ajoute :

«Il est probable que les Dag Rali supposaient que Moussa se déroberait et leur éviterait ainsi des frais en notre honneur, tout en laissant subsister le vague de la situation passée. Leur attitude est parfaite aujourd'hui et Doua assure qu'ils suivent Moussa et le parti de la paix depuis longtemps... "

Mais ce n'est pas seulement à propos de l'attitude à adopter face aux militaires français que Mûsa devra intervenir auprès des Dag-Ghâli. Il lui faudra, en effet, leur faire accepter comme aux autres groupes tributaires la nouvelle situation politique qu'instaure de fait la tournée Dinaux : les Kel-Ahaggar sont désormais des sujets soumis à la France et acceptent, signe tangible de cet état, de lui payer impôt. De plus, durant son séjour à Tamanrasset, Dinaux multipliera les signes qui rendent manifestes cette nouvelle donne. Tel est le cas de cette obligation faite aux Dag-Ghâli de lui vendre des chameaux de bât.

« Moussa paraît contrarié de ces demandes et cherche à obtenir que l'on patiente et que l'on passe en touristes dans le Hoggar. Je lui fais comprendre que je ne veux pas laisser de matériel en panne et que, si je n'avais pas besoin de chameaux, j'en demanderais quand même aux Dag Rali. Il faut qu'ils confirment 
leur soumission par des actes et il ne faut surtout pas qu'il y ait de mot d'ordre pour éviter tout rapport avec nous. [...] Moussa paraît craindre qu'un mécontentement des tribus amène un nouvel exode vers les Azdjer [...] mais il s'emploiera de son mieux à nous seconder. "

Ainsi tout laisse supposer que c'est bien contraints et forcés que les Dag-Ghâli, sur les conseils de Mûsa, acceptèrent les propositions de Dinaux. Dès lors, il peut être compréhensible que Mûsa ait au moins tenté d'éluder une autre demande de l'officier français : celle d'accepter l'installation de Foucauld à Tamanrasset au cœur même du territoire Dag-Ghâli. Dans le journal de Dinaux, journal qui se clôt le 04-09-1905, Foucauld a encore droit à une notation à la date du 19 août :

"Le Père de Foucauld commence les fondations de son ermitage sur la rive gauche de l'oued Tamanrasset à 3 ou 400 mètres des zéribas. Un puits creusé sur l'autre berge lui permettra de cultiver un petit jardin et la difficulté de l'eau se trouve ainsi tournée. Moussa s'est employé avec beaucoup de bonne volonté à réunir les travailleurs et aider au choix de l'emplacement. »

Parce qu'à cette date le principe de l'installation de Foucauld est désormais «admis ", cet extrait présente moins d'intérêt pour l'étude présente. On relèvera cependant qu'en ce qui concerne la datation des faits ce passage se trouve confirmé par le diaire de Foucauld. À la même date du 19 août, ce dernier note (Foucauld 1986: 49): "Commencé à construire chapelle. J'aurai une maison en pierre servant d'Eglise et de Sacristie, et une hutte en paille servant de dortoir, réfectoire, cuisine, parloir, chambre d'hôte... "

Par contre, ces deux extraits peuvent sembler en contradiction avec une lettre expédiée à Mme de Bondy le 13 août 1905. Lettre dans laquelle Foucauld précise (Foucauld $1966: 139$ ) : «Je commence demain à construire ici ma cabane. » Cette différence de cinq jours peut-elle s'expliquer par des réticences exprimées par Mûsa comme semblerait l'indiquer le passage (16 août 1905) précédemment cité du journal de Dinaux ? Ou bien n'est-elle due qu'à des problèmes d'ordre " technique » comme nous l'a suggéré Antoine Chatelard (in litt., 14 mars 1997) : «En fait il ( $=$ Foucauld) a dû commencer sur la rive droite au Nord et Mousa l'a fait recommencer sur la rive gauche si bien qu'il bénit la $1^{\circ}$ pierre seulement le 19. $)^{14}$

Quoiqu'il en soit, avec cet extrait daté du 19 août prend fin la série de notations concernant le Père de Foucauld dans le journal de route de Dinaux. Si nous avons tenu à les citer ici dans leur intégralité, c'est bien parce qu'au-delà de renseignements factuels, ces extraits nous obligent à revenir quelque peu sur ce moment crucial que fut l'installation de

\footnotetext{
${ }^{14}$ Dans une autre correspondance (16-05-1997), Antoine Chatelard nous a précisé que Paul Embarek, le domestique de Foucauld, se montrait parfaitement explicite sur ce point chronologique.
} 
Foucauld dans l'Ahaggar ${ }^{15}$. Celle-ci ne nous semble pas avoir été aussi simple et rapide que veut bien le dire la littérature hagiographique. Lors même le choix de Tamanrasset opéré, Mûsa a encore tenté d'influer sur la décision de Foucauld en ce qui concerne le lieu d'élection de son ermitage. Or, ce ne sont pas là de simples péripéties sans importance.

\section{L'ECHEC DE 1904}

Si l'établissement de Foucauld n'a pas été une évidence aussitôt acceptée c'est bien que, dans le contexte de l'époque, cette installation d'un religieux français et chrétien au cœur du Sahara posait problème. Dès lors c'est moins du seul bon vouloir de Mûsa que du rapport de force alors instauré entre les autorités militaires françaises et les Kel-Ahaggar qu'elle a dépendu. Durant les tournées qu'il effectua en 1904 avec Laperrine puis Roussel, Foucauld avait déjà le projet de s'établir dans l'Ahaggar. Désir qui concordait parfaitement avec les vues de Laperrine $^{16}$. Dès le 3 février 1904, ce dernier s'en confiait à Cauvet (AOM 14X2):

«De Foucauld est ici, il travaille le Touareg avec Ahmadou l'Ahl Azzi ; il partira s'installer la semaine prochaine à Akabli pour se perfectionner. Puis je verrai de le lâcher si tout a l'air de bien s'arranger; mais je voudrais qu'il puisse parler en Touareg avec les purs sans avoir à recourir à un Arabe. Ce ne serait pas banal d'avoir un ermite du Mont Koudiat Hoggar ou même de Tadjemout. " ${ }^{19}$

15 Bourgeot (1989) a justement fait remarquer qu'entre août 1905 et janvier 1907 , Foucauld a partagé son existence entre Beni Abbès, Tamanrasset et tournées dites d'《approvisement». Ce n'est qu'à partir de 1907 qu'il se fixc véritablement dans l'Ahaggar. Néanmoins, dans la mesure où installation n'est pas obligatoirement synonyme de résidence permanente, il nous semble que c'est bien à partir d'août 1905 , avec la construction de son ermitage à Tamanrasset, que l'on peut parler d'installation dans l'Ahaggar.

${ }^{16} \mathrm{La}$ teneur des relations alors existantes entre Laperrine et Foucauld mériterait à elle seule une étude approfondie. H. Didier nous semble cependant avoir pointé l'essentiel (1993 : 114): "Le ton chaleureux du courrier échangé entre Laperrine et Foucauld ne doit pas faire illusion: la sincérité de l'amitié qui les lie n'empêche pas l'existence d'un contrat politique entre eux: je te fais venir, je t'aide, si tu $m$ 'aides. "Voir également Casajus (à paraître) qui écrit que, du point de vue politico-militaire, le rôle de Foucauld « aura peu ou prou correspondu, au bout du compte, à celui que Laperrine lui destinait $»$.

${ }_{17}$ Dans une autre correspondance adressée à Cauvet le 10-02-1904 - et non en juin 1904 comme l'écrit Castillon du Perron (1982: 354) - figurent ces lignes parfois partiellement citées dans les biographies de Foucauld (AOM 14X2) : "Puisque Billou a navigué dans le Sahara je vais y lâcher de Foucauld il étudie le Touareg, a des médicaments et deux mehara pour se déplacer, vois tu si le Comte de Foucauld ex Hussard ex explorateur, trappiste en rupture de banc devient chapelain de Moussa ou d'un autre de même poil ce ne sera pas banal. " Voir également la lettre adressée par 
On sait qu'il n'en fut rien. Après son long périple de 1904 au Sahara central, Foucauld reprit le chemin de son ermitage de Beni Abbès. Malgré son désir, malgré l'accord initial de Laperrine, il ne put cette année-là s'installer dans l'Ahaggar. Sur ce point, l'abondante littérature consacrée à Foucauld est finalement peu prolixe.

Parfois aucune précision n'est fournie sur cet échec (Six 1962), parfois les causes en sont considérablement minimisées ${ }^{18}$. Mais le plus souvent, il est avancé que la situation n'étant pas clarifiée et Musa se dérobant devant la colonne conduite par Laperrine, c'est ce dernier qui n'autorisa pas Foucauld à s'établir dans l'Ahaggar ${ }^{19}$. Il est vrai qu'à des moments précis, notamment à Abalessa et Tit, Laperrine refusa son autorisation à Foucauld, comme le révèle le diaire de ce dernier. Raisons invoquées: l'absence de Mûsa et l'éventuelle opposition de "certains français » (Foucauld 1993, 106 et 111):

-Abalessa (22 mai 1904) «Le commandant Laperrine me laisserait ici s'il voyait Moussa ag Amastân, amenoukal des Hoggar, qui lui promet sa visite depuis un mois, est attendu par nous chaque jour, et n'est pas encore arrivé [...] Si Moussa ne nous rejoint pas, il ne veut pas me laisser au Hoggar, y trouvant trop de dangers. "

-Tit (28 mai 1904) «Moins par crainte des Touareg que par crainte de certains français, Laperrine ne m'autorise pas présentement à rester à Tit, ni ailleurs au Hoggar, plus tard on verra. "

Dans les deux cas, sans nier que ces causes aient pu influencer la décision de Laperrine, nous pensons cependant qu'il s'agit là de raisons secondaires. Concernant l'opposition éventuelle de "certains français », tout semble indiquer que c'est le capitaine Métois, alors chef de l'annexe d'In Salah, qui est ici visé. Très certainement à tort d'ailleurs. Bien qu'appartenant à des univers idéologiques opposés, Foucauld et Métois avaient su s'apprécier à leur juste valeur et leurs rapports n'étaient point conflictuels $^{20}$. Quoiqu'il en soit, même s'il ignorait les relations cordiales existant entre ces deux hommes, il est peu vraisemblable que Laperrine ait attendu mai 1904 pour découvrir qu'un éventuel antagonisme pouvait surgir entre Métois et Foucauld.

Plus intéressante nous apparaît la seconde raison invoquée : la non rencontre avec Mûsa. Car, au-delà de la personne même du chef KelAhaggar, cette absence de Mûsa, ses dérobades pour ne pas entrer en

Laperrine au commandant Regnault (in Gorrée 1946, II : 61).

18 Ainsi, dans sa belle biographie de Foucauld, H. Didier se contente d'écrire : «Un concours de circonstances retarda l'installation de Charles parmi les Touaregs » (1993: $116)$.

${ }^{19}$ Voir notamment Bazin (1931: 292), Carrouges (1963: 211) et Castillon du Perron (1982: 353).

${ }^{20}$ Lehuraux avait déjà bien relevé ce fait (1946:54). Voir également Suremain (1994) et Pandolfi (1997). 
contact avec Laperrine, sont un signe parmi d'autres de la situation qui régnait alors dans l'Ahaggar. C'est là un point fondamental sur lequel nous reviendrons. Mais il faut également relever que malgré cette absence de Mûsa la question d'une éventuelle installation de Foucauld a quand même été évoquée avec d'autres notables Kel-Ahaggar.

Ainsi, par un extrait déjà cité (voir supra) du journal de route de Dinaux on apprend qu'en mai 1904 Laperrine avait discuté de ce point avec Sughi et que c'est ce dernier qui lui avait conseillé de remettre à plus tard l'exécution de ce projet. Or, Sughi n'était autre alors que le représentant direct de Mûsa. Dans son diaire, à la date du 23 mai 1904, Foucauld évoque cette visite de Sughi et la possibilité ainsi offerte à Laperrine de négocier avec ce dernier son établissement dans l'Ahaggar. Mais il ne mentionne pas la réponse négative de Sughi (Foucauld 1993 : 108):

« Le Ct Laperrine reçoit aujourd'hui la visite de deux notables Kel Rela : Souriri, (30 ans, très proche parent de Mousa ag Amastân, désigné par lui comme son successeur à l'amenoukalat, frère de la jeune fille que Moussa aimait et qu'il n'a pu épouser) et Babba (45 ou 50 ans) venant à marches forcées de chez Mousa, porteurs d'une lettre de lui. La lettre dit que Mousa, au moment de se rendre près du Ct est tombé malade et qu'il délègue Souri avec pleins pouvoirs pour le remplacer et traiter de tout comme un autre lui-même. Si le Ct veut, il peut donc me laisser, Souri ayant tout pouvoir pour m'installer. "

La notation de Foucauld nous paraît fort significative. L'hypothèse formulée concerne la volonté du seul Laperrine mais non celle de Sughi et des Kel-Ahaggar. Or c'est pourtant bien là, nous semble-t-il, que résidait la question la plus épineuse. De même, en juillet-août 1904, le lieutenant Roussel avait formulé une demande similaire tant auprès de Sghir ag Bedda à Idelès qu'auprès de Si Mohammed ben Othman à Tazeruk $^{21}$. Dans les deux cas, comme le relève Foucauld (1993: 150151) la réponse fut négative. Or, ces trois hommes étaient des notables influents et des proches de Mûsa. Certes, en 1905, dans un contexte politique différent, ce dernier précisera à Dinaux que dès cette époque il a désavoué Sughi. Mais est-ce si sûr ? Quoiqu'il en soit, l'impression dominante reste qu'en 1904 les Kel-Ahaggar, y compris ceux qui suivaient Mûsa, étaient opposés à l'établissement du Père de Foucauld dans leur région. N'est-ce pas ce que reconnaît le principal intéressé dans une lettre adressée le 26-10-1905 à Mgr Livinhac (in Kergoat 1988, II : 128) : «L'année dernière, j'avais tenté à plusieurs reprises de m'installer

${ }^{21}$ Il est parfois avancé que Laperrine aurait demandé à Roussel, s'il rencontrait Mûsa, de négocier avec lui l'éventuelle installation de Foucauld (ex: Castillon du Perron 1982 : 354). En fait, la proposition de Laperrine était certainement bien plus large puisque Roussel formulera cette demande auprès de Sghir et de Sidi Mohammed et qu'il avait très certainement reçu l'accord de son supérieur pour entreprendre de telles démarches. 
au Hoggar, sans le pouvoir, par suite de l'esprit ombrageux des indigènes qui voyaient en moi un espion du gouvernement... »

On est alors en droit de se demander ce qui a permis que cette installation refusée en 1904 soit admise un an plus tard. Sans vouloir minimiser le rôle important qu'a pu alors jouer Mûsa, il nous paraît que seule la prise en compte de la nouvelle donne politique permet d'apporter quelques éléments de réponse.

\section{5 : UN CONTEXTE POLITIQUE NOUVEAU}

L'installation de Foucauld s'opère dans le même temps qu'est vraiment confirmée la soumission des Kel-Ahaggar aux autorités françaises. C'est dire que cet établissement, aussi important qu'il ait pu se révéler par la suite, n'était alors qu'un point secondaire par rapport à ceux qui, tant aux yeux des Kel-Ahaggar que des militaires français, s'avéraient fondamentaux. C'est d'ailleurs là le principal reproche que l'on peut adresser à la plupart des biographies consacrées au Père de Foucauld. La non prise en compte de la situation politique du Sahara central s'accompagne d'une survalorisation du rôle alors joué par des individus-clés comme Mûsa et Laperrine. S'il ne s'agit pas de nier l'influence de ces derniers, encore doit-on essayer de toujours la replacer dans ce contexte politique qui la permet et la légitime. Il nous faut donc, à grands traits, présenter la situation politique régnant alors dans l'Ahaggar ${ }^{22}$.

En janvier 1904, lors de sa rencontre avec Métois à In Salah, Mûsa signe un traité et accepte d'être nommé amenûkal par les autorités françaises. Vu l'ambiguïté de l'accord alors conclu, Mûsa peut présenter cette entrevue comme une négociation entre "égaux» et non comme une soumission. Malgré cette rencontre d'In Salah, rien n'est encore définitivement réglé. Comme le reconnaît Métois lui-même, In Salah ne constitue pas une soumission véritable. Nombre de points d'importance sont restés en suspens : perception de l'impôt, éventuelle installation française dans l'Ahaggar, problème des relations entre Kel-Ahaggar et Ifoghas... Aussi, en 1904, Mûsa peut encore se présenter aux siens comme celui qui a signé la paix avec les autorités françaises mais ceci sans représailles et sans capitulation puisque les Kel-Ahaggar ne se retrouvent pas en position de sujets soumis ${ }^{23}$. Dès lors, la politique de

\footnotetext{
${ }^{22}$ Pour de plus amples développements, nous nous permettons de renvoyer le lecteur à Pandolfi, à paraître.

${ }^{23}$ Dans l'introduction de son rapport de tournée, Dinaux présente ainsi la situation (1907a) : "Moussa s'était aussitôt retiré dans l'Adrar, où il habitait depuis plusieurs années; connu et estimé dans tout le Sahara pour sa générosité, sa droiture et sa bravoure, il put augmenter encore sa popularité en annonçant partout, depuis $R$ 'at et l'Aïr jusqu'aux postes du Niger, le pacte qu'il venait de conclure avec nous, et qu'il sut présenter comme un succès diplomatique, réservant l'indépendance des Touareg et
} 
Mûsa consistera à camper le plus longtemps possible sur cette position. À l'inverse, Laperrine n'aura de cesse de transformer cette négociation d'In Salah en une soumission en bonne et due forme. La teneur de la lettre envoyée par Dinaux à Mûsa le 3 avril 1905 est sur ce point on ne peut plus claire. Après s'être étonné des dérobades de Mûsa depuis la rencontre d'In Salah, l'officier français admoneste le chef Kel-Ahaggar (A.O.M. 22H68) :

« Comment! depuis plus d'un an que les autorités d'In Salah vous ont ouvert les bras et vous ont reçu comme un fils de la France, vous n'avez cherché qu'à vous éloigner et à augmenter la distance entre nous. Que craignez vous donc, et ne comprenez vous pas votre intérêt et celui de vos gens ?[...]

« Nous avons rouvert nos marchés à vos gens et nous les accueillons tous avec bienveillance. Mais comprenez bien qu'en faisant cela nous n'avons pas fait un traité entre deux puissances: nous l'avons fait parce que les Touareg sont maintenant les sujets du gouvernement français.

«Et vous, Moussa ag Amastane qui êtes parmi les plus sages et les plus influents, il faut absolument que vous compreniez cela et que vous le fassiez comprendre à vos gens.

«Et il faut aussi dans votre intérêt personnel que vous vous rapprochiez de nous et que vous soyez la main du gouvernement. Puissiez vous comprendre mes conseils et les suivre avec bonne volonté. ")

Dans un tel contexte, l'objectif premier et avoué de la tournée Dinaux, celle-là même qui amène Foucauld dans l'Ahaggar, était clairement fixé (Laperrine 1913: 405): «... il s'agissait de remettre au point la soumission de Moussa ag Amastane faite à In Salah et de bien établir que c'était une soumission et non une alliance. Il fallait faire accepter par les tribus un léger impôt qui fût une marque tangible de cette soumission. "

De toutes les tournées menées au Sahara central par les officiers français au début du siècle, celle conduite par Dinaux en 1905 est certainement la plus éloignée du modèle idéal de la tournée d'《apprivoisement» prôné par Laperrine. Il s'agit, avant tout, d'imposer - si nécessaire - leur soumission aux Kel-Ahaggar et pour ce faire de multiplier les marques manifestes de cette situation. Mûsa tentera bien de négocier au mieux cette nouvelle donne à laquelle il se trouve confronté. Mais, au final, il devra se plier à la ferme volonté politique des autorités françaises et reculer sur tous les points envisagés. Parmi eux, figure de fait la demande, formulée par Dinaux, d'une installation de Foucauld dans l'Ahaggar. Dès lors, on peut légitimement s'interroger sur cette «bonne volonté » de Mûsa si souvent mise en avant par les commentateurs. Et ce d'autant plus que même sur cet enjeu

leur garantissant le libre commerce. [...] Les Touareg voulaient bien reconnaître que Moussa avait "fait le bien et la paix" avec nous; mais ils ne se considéraient pas comme nos sujets. Il en résultait une situation ambiguë, fausse, peu flatteuse pour nous... » 
secondaire le chef Kel-Ahaggar a manifestement eu besoin de convaincre les siens.

Dans une telle situation, la notation idyllique de J.-F. Six, pour qui Foucauld s'avançait "désarmé ${ }^{24}$ nous paraît bien éloignée de la réalité. Bien au contraire, c'est par les militaires français qui, conséquence de la défaite subie par les Touaregs au combat de Tit (1902), signifient fermement aux Kel-Ahaggar leur état de sujets politiquement soumis que Foucauld est introduit et installé dans l'Ahaggar. L'accord obtenu n'est-il pas dès lors, à ce moment-là, que la constatation d'un état de fait : militairement défaits et politiquement soumis, que pouvaient faire d'autre Mûsa et les siens? D'ailleurs Foucauld, toujours plus lucide que la plupart de ses thuriféraires, ne s'y trompera pas. Dans une correspondance en date du 3 juillet 1904, il écrivait (Foucauld 1991 : 81):

"Les indigènes nous reçoivent bien : ce n'est pas sincère, ils cèdent à la nécessité. Combien de temps leur faudra-t-il pour avoir les sentiments qu'ils simulent ? Peut-être ne les auront-ils jamais. S'ils les ont un jour, ce sera le jour qu'ils deviendront chrétiens. Sauront-ils séparer entre les soldats et les prêtres, voir en nous des serviteurs de Dieu, ministres de paix et de charité, frères universels? Je ne sais. "

Devant de telles interrogations, les stéréotypes dans lesquels on enferme souvent le personnage apparaissent bien réducteurs. Exempter Foucauld de tout rapport avec l'avancée coloniale au Sahara tout comme ne voir en lui qu'un alibi spirituel du colonialisme français ne sont que les deux faces - en apparence opposées - d'une même vision simplificatrice. Replacer l'établissement de Foucauld dans son contexte réel, celui du rapport de force alors établi entre les autorités coloniales françaises et les Kel-Ahaggar, n'est à nos yeux que la reconnaissance d'un fait historique difficilement niable. Mais si Foucauld nous questionne encore aujourd'hui, c'est bien que, malgré cela, par son œuvre et sa vie, y compris dans l'impact qu'elle a pu avoir auprès de certains Touaregs (Casajus 1997), il a su et pu aller bien au-delà de ce point de départ.

\footnotetext{
${ }^{24}$ Évoquant le premier séjour (1904) de Foucauld dans l'Ahaggar, cet auteur écrit : "Frère Charles qui a vécu à Béni-Abbès entre la garnison française et la population arabe, descend maintenant vers le Sud à la rencontre des ennemis des Arabes. Il va vers eux en ami, lui qui est pour eux un étranger et un ami des Arabes. Il va vers eux désarmé " (Six, $1962: 131)$.
} 


\section{Bibliographie}

Documents d'archives

DINAUX, Cne. 1907a. "Rapport de tournée du Capitaine Dinaux, Chef de l'Annexe d'In Salah, Commandant de la compagnie des Oasis sahariennes du Tidikelt-Ahnet, Adrar nigritien, Ahaggar, Aïr septentrional (3mai/29octobre 1905) », A.O.M. 22H68.

- 1907b. "Carnet de renseignements politiques ", Appendice $n^{\circ}$ IV du rapport de tournée du Capitaine Dinaux, A.O.M. $22 \mathrm{H68}$.

LAPERRINE, H. Correspondance adressée à G. Cauvet, A.O.M.14X2.

ROUSSEL, Lieut. 1904. "Rapport de tournée du Lieutenant Commandant le $2^{\circ}$ Peloton de la compagnie du Tidikelt, TifedestAhaggar (28 mai-20 septembre 1904)», A.O.M. 22 H67.

Ouvrages et articles

AMBLER, J. S. 1966. The French Army in Politics, 1945-1962, Columbus, Ohio State University Press.

ANDERSON, B. 1996. L'imaginaire national. Réflexions sur l'origine et l'essor du nationalisme, Paris, La Découverte.

ANTIER, J.-J. 1997. Charles de Foucauld, Paris, Perrin.

BAZIN, R. 1931. Charles de Foucauld, explorateur du Maroc, ermite au Sahara, Paris, Plon.

BENHAZERA, M. 1908. Six mois chez les Touareg du Ahaggar, Alger, A. Jourdan.

BOURGEOT, A. 1989. "Le rôle politique de Charles de Foucauld au Sahara central : 1901-1916», Mondes en Développement XVII (65), p. 139-153.

CARROUGES, M. 1963. Charles de Foucauld, explorateur mystique, Paris, Union générale d'éditions.

CASAJUS, D. 1997. «Charles de Foucauld face aux Touaregs, Rencontre et malentendu ", Terrain 28, pp. 29-42.

- à paraître. "La vie saharienne et les "Vies" de Charles de Foucauld ", in L.Galand (éd.), Lettres au marabout. Messages touaregs au P. de Foucauld.

CASTILlON DU PERRON, M. 1982. Charles de Foucauld, Paris, Grasset.

CHATELARD, A. 1995. "Charles de Foucauld linguiste ou le savant malgré lui », Études et Documents berbères 13, pp. 145-177.

DERVIL, G. 1932. Trois grands Africains : Dans l'intimité de Lyautey, Laperrine, Foucauld, Paris, J. Suse.

DIDIER, H. 1993. Petite vie de Charles de Foucauld, Paris, Desclée de Brouwer. 
DINAUX, Cne. 1907c. « Rapport de tournée du capitaine Dinaux, chef de l'annexe d'In Salah, commandant de la compagnie des Oasis sahariennes du Tidikelt (3 mai-29 octobre 1905) », Bulletin du Comité de l'Afrique française-Renseignements coloniaux 1, pp. 11$19 ; 2$, pp. 42-51;3, pp. 62-72.

DINAUX, Gén. 1946. "L'installation du Père de Foucauld au Hoggar », Cahiers Ch. de Foucauld 3/4, pp. 35-43.

FOUCAULD, Ch. de. 1938. Lettres à Henry de Castries, Paris, Grasset.

- 1966. Lettres à Mme de Bondy, Paris, Desclée de Brouwer.

- 1986. Carnets de Tamanrasset (1905-1916), Paris, Nouvelle Cité.

- 1991. "Cette chère dernière place". Lettres à mes frères de la Trappe, Paris, Cerf.

- 1993. Carnet de Beni Abbès (1901-1905), Paris, Nouvelle Cité.

- 1994-1995. «Chez les Touaregs (Taïtoq, Iforas, Hoggar), MarsSeptembre 1904 ", Bulletin trimestriel des Amitiés Charles de Foucauld 115, pp. 9-18;116, pp. 6-13; 117 pp. $15-24 ; 118$ pp.815.

GAUTIER, E. F. 1906. «Du Touat au Niger», La Géographie XIII, pp. 5 - 18.

- 1931. Trois Héros: Le général Laperrine, le Père de Foucauld, Prince de la Paix, Paris, Payot.

GORREE, G. 1946. Les amitiés sahariennes du Père de Foucauld, Paris, Arthaud, 2 tomes.

- 1947. Sur les traces de Charles de Foucauld, Paris, Arthaud.

GUILLO-LOHAN, Lt. 1903. «Un contre-rezzou au Hoggar », Bulletin du Comité de l'Afrique Française-Renseignements coloniaux 13, pp. 205-214, 239-246 \& 257-267.

KERGOAT, L. 1979. "Les Poètes Touaregs », Revue des études islamiques XLVII (2), pp. 209-223.

- 1988. Charles de Foucauld et l'islam. Politique et mystique, Thèse d'état, Université de Paris-Sorbonne.

LAPERRINE, H. 1905. "Une tournée dans le sud de l'annexe du Tidikelt (4 mars-30 juillet 1904) », Bulletin du Comité de l'Afrique françaiseRenseignements coloniaux 2, pp. 37-47.

- 1913. "La pacification des Touaregs: Notes et Souvenirs", Questions diplomatiques et coloniales 35, pp. 393-407, 517-529 \& 651-659; 36 pp. 74-81 \& 137-146.

LEHURAUX, L. 1946. Au Sahara avec le Père Charles de Foucauld, Paris, Éditions St Paul.

LENCLUD, G. 1991. "Le monde selon Sahlins », Gradhiva 9, pp.49-62.

LESOURD, P. 1933. La vraie figure du Père de Foucauld, Paris, Flammarion. 
MARTY, P. 1920. Etudes sur l'Islam et les tribus du Soudan, Paris, Leroux, 3 tomes.

MERAD, A. 1976. Charles de Foucauld au regard de l'Islam, Lyon, Chalet.

METOIS, F.A. 1906. La soumission des Touaregs du Nord, Paris, Challamel.

MOREL, M.H. 1947. "Remarques sur la vie mentale et les gestes des Touareg de l'Ahaggar", Travaux de l'Institut de Recherches Sahariennes IV, pp. 127-144.

PANDOLFI, P. 1997. Touaregs de l'Ahaggar. Parenté et résidence chez les Dag-Ghali, Paris, Karthala.

- 1998 (à paraître). "In Salah 1904-Tamanrasset 1905 : La double soumission des Kel-Ahaggar », Cahiers d'études africaines 148.

RICHER, A. 1924. Les Oulliminden, Paris, Larose.

SAHLINS, M. 1989. Des îles dans l'histoire, Paris, Hautes Etudes, Gallimard-Le Seuil.

SIX, J.-F. 1962. Vie de Charles de Foucauld, Paris, Seuil.

SUREMAIN, M. de. 1994. "Portraits », Bulletin des Amitiés Charles de Foucauld 116, pp. 19-22.

SERPETTE, M. 1997. Foucauld au désert, Paris, Desclée de Brouwer.

VIGNAUD, J. 1943. Frère Charles ou la vie hérö̈que de Charles de Foucauld, Paris, Albin Michel. 